\section{Análise do processo de treinamento de uma universidade corporativa pela perspectiva da abordagem sistêmica de treinamento*}

Rodrigo Menezes Fernandes ${ }^{1}$ Janduhy Camilo Passos ${ }^{2}$ Márcia Freire de Oliveira ${ }^{3}$ Ismael Barbosa ${ }^{4}$ Henrique Geraldo Rodrigues 5

* Recebido em: 17/09/2015.

Aprovado em: 21/03/2017.

1 Graduado em administração, pela Universidade Federal de Uberlândia.

2 Doutor em Administração de empresas pela Fundação Getúlio Vargas (FGV/SP). É professor da Faculdade de Gestão e Negócios da Universidade Federal de Uberlândia e atua nas áreas de gestão de pessoas, comportamento organizacional, docência em administração e psicologia organizacional. E-mail: camilo@ufu.br.

3 Professora da Faculdade de Gestão e Negócios da UFU, pós-doutorado em Administração pela Faculdade de Economia e Administração e Contabilidade de Ribeirão Preto da USP, doutorado em Engenharia de Produção pela UFSCar, mestrado em Engenharia de Produção pela EESC-USP, graduação em Administração pela UFU. Atua principalmente com os seguintes temas de pesquisa: gestão de pequenas empresas e empresas familiares, inovação, empreendedorismo e aglomerações produtivas. E-mail: marciafreire@ufu.br.

4 Graduação e mestrado em Ciências Contábeis, pela Universidade Federal de Uberlândia, e graduação em administração pública, pela Universidade Federal de Ouro Preto. Atua como professor do ensino superior nas áreas de Ciências Contábeis e Administração. E-mail: barbosamg@yahoo.com.br.

Doutorado em administração de empresas pela Universidade Presbiteriana Mackenzie. Atua como professor na Faculdade de Gestão e Negócios, da Universidade Federal de Uberlândia. E-mail: henrique@ufu.br.

\section{Analysis of the training process of a corporate university through the perspective of the systematic approach to training}

\section{Resumo}

Objetivou-se, neste trabalho, analisar o processo de treinamento desenvolvido pela universidade corporativa de uma indústria desenvolvedora de softwares de gestão empresarial, com o propósito de discutir de que forma o processo de treinamento realizado na universidade corporativa pesquisada contempla elementos e características da abordagem sistêmica de treinamento. A estratégia de pesquisa adotada foi o estudo qualitativo básico e os dados foram coletados por meio de entrevistas qualitativas individuais semiestruturadas com gestores e levantamento documental estruturado. Os resultados mostram que a adoção de elementos da abordagem sistêmica de treinamento pela universidade corporativa estudada contribui para o desenvolvimento de ações de capacitação que proporcionam aprendizados efetivos aos seus participantes e alinhados aos objetivos estratégicos da organização. Não obstante, por estarem, fortemente, baseados na modalidade de educação a distância, os programas de treinamento da universidade corporativa contribuem mais para a aquisição de aprendizados técnico-funcionais e menos para o desenvolvimento de aprendizados comportamentais pelos participantes. Pondera-se que tal resultado seja decorrente, em parte, das limitações à interação e cooperação entre os participantes dos treinamentos, quando este é realizado por meio da modalidade de educação a distância. Como principal limitação, salienta-se que os resultados da pesquisa não podem ser generalizados, dado que se baseiam, sobremaneira, na visão dos gestores entrevistados, cuja organização foi selecionada por conveniência. A pesquisa contribui para ampliar as discussões sobre as abordagens de treinamento utilizadas no âmbito da educação corporativa e seus resultados. Palavras-chave: Gestão do treinamento. Abordagem sistêmica de treinamento. Universidades corporativas.

\begin{abstract}
The objective behind this work is to analyze the training process developed by the Corporate University of a software development industry in business management. The goal is directed towards discussing in which manner the training process realized in the Corporate University at the core of this study, contemplates elements and characteristics of the systemic training approach. The research strategy adopted was the basic qualitative study, where the data were collected by means of semi-structured individual qualitative interviews and structured document
\end{abstract}


surveys. The results showed that the adoption by the Corporate University under focus, of elements from the system approach to training, contributed to training activities that provide effective learning for its participants and which are aligned with the organizations strategic objectives. These programs are strongly centered upon the modality of learning at a distance yet, these training programs from the Corporate University contributed more towards the acquisition of technical and functional learning on the part of the participants, at the expense of behavioral learning. A thought that comes to mind is that such a result is due in part to the limitations found in interaction and cooperation between participants of the training, especially when it is performed through a learning process at a distance regime. As the principal limitation, one can highlight that the results from the research cannot be generalized as they are based, excessively, on the view of the interviewed managers, whose organization was selected purely for convenience sake. The research contributed to extend the discussions concerning the training approaches used in the corporative educational environment along with their results.

Keywords: Management training. Systemic approach to training. Corporate Universities.

\section{Introdução}

Atualmente, em virtude do ambiente de negócios marcado pela constante inovação, competitividade e necessidade de diferenciação ante a concorrência, acredita-se ser imprescindível o desenvolvimento, nas organizações, de mecanismos para a geração, disseminação e aplicação do conhecimento focado nas necessidades do negócio e alinhado às estratégias organizacionais. Nessa linha, o conteúdo do conhecimento requisitado das pessoas altera-se com uma frequência cada vez mais intensa, o que faz dos processos de treinamento e desenvolvimento um elemento de importância especial para o desempenho das organizações (MUNDIM, 2002).

$\mathrm{Na}$ verdade, considera-se que tal mudança teve início na década de 1990, em decorrência das dificuldades enfrentadas pelas áreas de recursos humanos em relação ao atendimento às demandas estratégicas da organização. Além de serem pautados por respostas reativas (ou treinamentos reativos), os processos de treinamento focavam, normalmente, apenas, o desenvolvimento de habilidades dos funcionários, sendo pouco inovadores quanto às formas de disseminação e gerenciamento do conhecimento (ALPERSTEDT, 2001; MUNDIM, 2002).

Conforme aponta Silva (2006), as vantagens competitivas das organizações estão relacionadas à integração dos programas de treinamento com a estratégia organizacional, pois os resultados dos programas de treinamento afetam a produtividade dos empregados, contribuindo para o aumento da eficiência empresarial e a redução de custos. Todavia, é preciso que exista um sistema constante de planejamento e avaliação das ações de treinamento, com a finalidade de assegurar o retorno adequado do investimento realizado e ampliar o alcance dos objetivos a que essas ações educativas se destinam (MILKOVICH; BOUDREAU, 2000).

Nessa perspectiva, o surgimento das universidades corporativas (UCs) trouxe uma alteração significativa para a prática do treinamento, pois elas se constituíram, também, em laboratórios de aprendizagem permanente para a organização. Tal aspecto é relevante, considerando que, no contexto da economia global do conhecimento, a atualização do conhecimento e das capacidades das pessoas é vista como um processo contínuo, que se volta não apenas aos empregados, mas também aos fornecedores, clientes e grupos específicos da comunidade em que a organização encontra-se inserida (MEISTER, 1999).

Não obstante, se reconhece que não é uma tarefa fácil induzir a melhoria do desempenho no trabalho mediante a promoção de treinamentos, por meio das UCs. Estudos empíricos mostram que a participação em treinamentos promovidos por uma UC nem sempre leva a efeitos significativos sobre o desempenho funcional das pessoas (MORIN; RENAUD, 2004; SHAM, 2007). Os resultados destes sugerem, em especial, o entendimento de que os processos de treinamento e desenvolvimento precisam ser readequados ao contexto da UC, levando-se em conta tanto aspectos processuais do planejamento e execução da capacitação, quanto os relativos ao alinhamento das ações da UC às estratégias da organização que abriga a UC.

Julga-se que, dentre as diversas abordagens à prática do treinamento nas organizações, a abordagem sistêmica contenha elementos que conferem uma maior atenção às necessidades de desenvolvimento, efetivamente, apresentadas pelo indivíduo, ao mesmo tempo em que toma o processo de treinamento como um sistema afetado pela dinâmica do contexto organizacional, requerendo que os objetivos de treinamento estejam relacionados 
aos objetivos organizacionais, bem como em consonância com a estratégia organizacional (SILVA, 2006).

Desse modo, levando-se em consideração que o processo de treinamento se mostra um aspecto crítico para o alcance dos propósitos de uma UC e que se tem em conta que é possível lançar mão de diferentes abordagens para a prática do treinamento nas organizações, objetivou-se, neste trabalho, analisar o processo de treinamento desenvolvido pela UC de uma indústria desenvolvedora de softwares de gestão empresarial. Com base no exposto, apresenta-se a seguinte questão de pesquisa: como o processo de treinamento realizado na UC pesquisada contempla elementos e características da abordagem sistêmica de treinamento?

Além desta introdução, o artigo é composto pelas seguintes partes: o referencial teórico, que faz breves considerações a respeito do treinamento nas organizações, apresenta a abordagem sistêmica de treinamento e versa sobre a UC; aspectos metodológicos; apresentação e discussão dos resultados, e considerações finais.

\section{Referencial teórico}

\subsection{Pressupostos básicos sobre a gestão do treina- mento nas organizações}

De modo geral, o treinamento é compreendido como a educação prática que favorece o domínio de uma habilidade ou de um cargo (KRUMM, 2005). Logo, conforme indica Carvalho (2007), tem por finalidade fornecer suporte às pessoas na organização, em sua busca por eficiência, mediante o uso de métodos e técnicas apropriadas.

Em um enfoque multidisciplinar, dada as múltiplas interações humanas com os seus grupos de referência (lar, escola e trabalho, dentre outros), o treinamento caracteriza-se como um meio de educação voltado para o trabalho, pois seu intuito consiste em preparar o indivíduo para o desempenho funcional pleno (CARVALHO, 2007). Além disso, o treinamento se interliga à educação, uma vez que treinar implica despertar aptidões e capacidades latentes, configurando-se, assim, como um dos recursos para o desenvolvimento pessoal (CARVALHO; NASCIMENTO; SERAFIM, 2012).

Isso significa, também, que o treinamento toma parte no desenvolvimento de habilidades, atitudes e novos conceitos pelas pessoas, pois a aprendizagem efetua mudanças no comportamento destas, originando novos hábitos, conhecimentos, atitudes e destrezas (KRUMM, 2005; BOOG, 2007).

No contexto organizacional, espera-se que o treinamento contribua para o desenvolvimento das pessoas, naqueles elementos pertinentes à sua capacitação pessoal, aspirações e motivações, além de auxiliar na adaptação do indivíduo ao ambiente organizacional. Portanto, se considera que o processo de treinamento deva estar configurado de forma integrada aos demais sistemas da organização, em especial o de gestão de pessoas.

Nessa ótica, o planejamento e o desenvolvimento do treinamento devem focar os objetivos organizacionais, o desenvolvimento tecnológico e os objetivos pessoais (BOOG, 2007). Em relação aos objetivos organizacionais, a programação do treinamento deve contemplar o tempo disponível (curto, médio ou longo prazo), e a forma desejada (integração, qualificação profissional, aperfeiçoamento, especialização ou formação).

$\mathrm{O}$ atendimento ao desenvolvimento tecnológico, por sua vez, decorre por meio de programas de treinamento que, em função do crescimento e da acumulação do capital tecnológico, buscam prover a atualização das pessoas que atuam na organização, para que não ocorra a obsolescência de seu conhecimento.

As ações de treinamento, também, devem atentar para adequar os efeitos das mudanças que ocorrem dentro e fora do ambiente empresarial às necessidades das pessoas. Portanto, passa a ser um dispositivo para que o empregado alcance por meio do trabalho as suas metas de realização pessoal e profissional (BOOG, 2007).

Quanto às vantagens para as organizações, é interessante destacar que o treinamento permite que as pessoas, que nelas atuam, tenham uma visão mais ampla e de longo prazo. Por meio das ações educativas, fomenta-se uma aprendizagem contínua visando desenvolver atitudes, forma de pensamento, hábitos, competências e uma visão de negócio que possam dotar as pessoas de capacidades mentais que aperfeiçoem o trabalho que executam (TOLEDO; MILONI, 1986).

Ainda, é preciso considerar que algumas formas de treinamento possibilitam, também, transmitir às pessoas recém-contratadas elementos culturais considerados relevantes pela organização. Os primeiros contatos que se estabelecem com o novo funcionário são verdadeiramente decisivos à sua vida profissional. Por isso, salienta-se a importância de que os treinamentos sejam planejados, conduzidos e administrados de forma atenta, tendo em 
vista que eles incidem sobre a fixação das primeiras impressões recebidas, consolidando atitudes das pessoas, sejam elas positivas ou negativas.

Nas organizações, são diversas as situações que desencadeiam as necessidades de treinamentos, dentre as quais se destacam: a existência de recém-formados; os cargos e suas funções que se modificam continuamente, originando necessidade de reciclagem profissional; empregados novos; conflitos internos e problemas interpessoais entre chefes e subordinados, e a necessidade de empregados multifuncionais. Ademais, espera-se que as ações de treinamento auxiliem na solução de problemas organizacionais, como os relacionados a absenteísmo, desperdícios de materiais e retrabalhos, redução da capacidade produtiva, ausência de motivação, atendimento de forma negligente e danos patrimoniais (BOOG, 2007; CARVALHO; NASCIMENTO; SERAFIM, 2012; TOLEDO; MILONI, 1986).

Desse modo, as ações de treinamento podem trazer vantagens à empresa, envolvendo o aumento de produtividade, a redução de custos e a melhoria da qualidade do produto, as quais, se argumenta, contribuem para a elevação do desempenho e resultados corporativos (BOOG, 2007; TOLEDO; MILONI, 1986).

\subsection{Sobre as universidades corporativas: origem e pressupostos centrais}

Embora os programas educacionais sempre tenham existido nas organizações, normalmente eram restritos aos níveis de média e alta gerência, pois, para as pessoas alocadas nos níveis operacionais, normalmente eram direcionadas ações de treinamento voltadas para aspectos específicos do trabalho (EBOLI, 2002).

Acredita-se que, a partir do final dos anos 1980, a disseminação das UCs como estratégia de gerenciamento para o desenvolvimento de profissionais tenha colaborado, em especial, para que os tradicionais programas de treinamento assumissem uma conotação mais abrangente, voltada à disseminação da educação nos diversos níveis de todos os funcionários da organização, além de cooperar para a eficácia da gestão empresarial (BRANDÃO, 2006; HOLLAND; PYMAN, 2006).

No Brasil, a difusão do conceito de UC deu-se iniciou-se na década de 1990, em decorrência da globalização dos mercados e de seus efeitos sobre as organizações, as quais se viram impelidas a investir de modo sistêmico na qualificação de seus empregados e a com- prometer-se com seu desenvolvimento contínuo. Nessa fase, observam-se como primeiras experiências de UCs as iniciativas da Academia Accor (1992), a Universidade Martins do Varejo (1994) e a Universidade Brahma (1995), que, juntamente a outras, totalizavam dez UCs no País (BRANDÃO, 2006; EBOLI, 2002).

A partir dos anos 2000, o crescimento das UCs no Brasil tornou-se expressivo, sendo capitaneado pelos mesmos fatores que impulsionaram a proliferação dessas organizações no mundo, a saber: as organizações flexíveis e enxutas; a era do conhecimento; a rápida obsolescência do conhecimento associado ao sentido de urgência; a empregabilidade, que propunha a noção de emprego para a vida toda, em vez do emprego para toda a vida e educação para estratégia global, com perspectiva internacional dos negócios (BRANDÃO, 2006; EBOLI, 2002).

Meister (1999) considera que o principal objetivo de uma UC seja o de preparar as pessoas da organização para obter vantagens diante das mudanças emergentes e institucionalizar uma cultura de aprendizagem contínua, alinhada às estratégias centrais do negócio. Por isso, tem-se que o modelo de UC deve basear-se em competências e conectar a aprendizagem com as necessidades estratégicas do negócio. Pela perspectiva da UC, o aprendizado como um valor e uma atividade contínua no cotidiano das empresas, visando à formação de uma força de trabalho possuidora de qualidade.

Nesse sentido, no contexto de uma UC, são instituídos programas de capacitação permanentes, alinhados aos objetivos da organização, que fomentam uma linguagem comum e conduzem a obtenção de resultados organizacionais (MEISTER, 1999). Como a UC centraliza o gerenciamento estratégico da educação e do treinamento, pode ser tomada por uma função pertinente a gestão de pessoas, não se configurando como uma unidade ou órgão empresarial à parte.

Conforme sugere Eboli (2002), a adoção da filosofia de uma UC pressupõe a consideração dos seguintes pressupostos no contexto organizacional:

a) busca-se o desenvolvimento das competências críticas do negócio e de sua competitividade, em vez, apenas, das habilidades individuais;

b) privilegia-se o aprendizado organizacional fortalecendo a cultura corporativa e o conhecimento coletivo, e não apenas o conhecimento individual;

c) tem-se por foco as necessidades do negócio, 
tornando o escopo estratégico em vez de focado, exclusivamente, nas necessidades individuais;

d) a concepção e desenho das ações e programas educacionais ocorrem a partir das estratégias de negócios;

e) adota-se o conceito de educação inclusiva, com base na qual se busca desenvolver competências críticas no público interno e externo, e não somente nos empregados;

f) contempla-se a possibilidade de ser um projeto virtual e não necessariamente um local físico.

Como se observa, a prática relativa às UCs trata-se de uma ação consonante as estratégias da empresa, além de constituir-se em um teste de aprendizagem para a organização e um instrumento de educação permanente, dado que as UCs objetivam, também, desenvolver o modo de ser, pensar e fazer dos mais diversos públicos e categorias profissionais com os quais a organização interage (MEISTER, 1999; EBOLI 2002).

\section{Metodologia}

Este estudo é de natureza qualitativa e classifica-se, quanto aos seus objetivos, como descritivo. Isto representa que se buscou compreender o objeto de estudo ? o processo de treinamento aplicado em uma UC - por meio da descrição de seus elementos, mecanismos e características, com a finalidade de apreender informações contextuais necessárias à explicação do problema (SANTOS, 1999).

A pesquisa foi realizada juntamente à universidade corporativa (denominada UCX, com o propósito de manter o anonimato da organização pesquisa pesquisada) de uma empresa de grande porte do segmento de tecnologia da informação (identificada, neste trabalho, como Empresa X). Tendo em vista que as UCs são consideradas uma importante dimensão dos sistemas de gestão de pessoas nas organizações, a escolha da UCX foi orientada pelo fato de a Empresa X constar em diversas edições, dos últimos dez anos, do ranking das Melhores Empresas para Trabalhar no Brasil, conforme o Great Place to Work.

Como estratégia de pesquisa, adotou-se a abordagem do estudo qualitativo básico (MERRIAM, 2002), o qual envolve, esclarece Creswell (2010), uma forma genérica de análise, em que os dados qualitativos são analisados e relatados mediante alguns poucos temas ou perspectivas. Assim, na coleta dos dados usou-se a entrevista qualitativa individual semiestruturada (BRYMAN, 2004) e o levantamento documental estruturado (FLICK, 2009).
Os dados da pesquisa foram coletados no ano de 2013, por meio de entrevista com dois gestores da UCX e levantamento de documentos nos arquivos físicos e digitais da organização.

Mediante a transcrição dos dados, a análise dos dados deu-se em consonância com a proposta do estudo qualitativo básico, pelo que se buscou descrever o fenômeno mediante a visão dos próprios sujeitos, em consonância com interpretação que fazem de suas experiências e os significados que atribuem a elas (MERRIAM, 2002).

\section{Apresentação e análise dos resultados}

\subsection{Histórico, objetivos e estrutura da UCX}

A UCX foi criada em 2004, como uma unidade organizacional de uma empresa brasileira de grande porte desenvolvedora de softwares de gestão empresarial conhecidos por enterprise resource planning (ERP). No entendimento dos gestores da UCX, ao implantar essa nova unidade, a Empresa $\mathrm{X}$ buscava, principalmente, uma forma de suprir sua demanda por profissionais aptos a atender as organizações clientes em suas necessidades de melhoria dos processos de gestão e operacionais, via implementação do ERP.

Considera-se que o alinhamento da UCX à estratégia da Empresa X mostra-se por meio de seus objetivos, dentre os quais se destacam: criar e manter modelos de treinamento e certificação que levem a melhorias e inovações no negócio; treinar grande quantidade de colaboradores no menor tempo possível; aumentar o grau de absorção do conhecimento pelo colaborador; aumentar os níveis de produtividade dos colaboradores; atender os clientes com qualidade no serviço prestado; recompensar os colaboradores com base no nível de competência; apoiar os processos de seleção interna, com informações sobre o nível de conhecimento técnico dos colaboradores; realizar parcerias com universidades para ofertar treinamentos à comunidade, com o objetivo de aumentar a empregabilidade das pessoas.

A estrutura organizacional da UCX é funcional, estabelecida em torno dos cargos existentes, quais sejam os de desenvolvedor de e-learning, analista de documentação, consultor, analista de treinamento, consultor e assistente administrativo, além da coordenação geral.

Em relação aos processos operacionais da UCX, os processos-chave são os de formalização de produtos e certificação de colaboradores e clientes. O de formaliza- 
ção de produtos envolve a criação de cursos de educação à distância $(\mathrm{EaD})$, elaboração e revisão de conteúdo $\mathrm{EaD}$, documentação do sistema, criação e manutenção de materiais didáticos.

O processo de certificação de colaboradores e clientes, por sua vez, compreende o planejamento, organização, acompanhamento e avaliação de treinamentos presenciais e certificação de colaboradores. As certificações pela UCX significam que os colaboradores responsáveis pelas consultorias de implantação do ERP nos clientes da Empresa X e pela monitoria e suporte ao cliente após a implantação, estão capacitados e preparados para executar suas tarefas. São certificadas, também, as pessoas que, nos clientes, farão uso dos sistemas implantados.

\subsection{A análise do processo de treinamento da UCX pela visão da abordagem sistêmica de treinamento}

Nesta seção, em consonância com o objetivo proposto para o trabalho, é feita a descrição e a análise do processo de treinamento da UCX, sob a perspectiva das etapas que compõem a abordagem sistêmica de treinamento apontada por Snell e Bohlander (2009). Tais etapas são: levantamento de necessidades de treinamento, elaboração do projeto de treinamento, definição dos métodos de treinamento, implementação e avaliação do projeto.

\subsubsection{Levantamento das necessidades de treinamento}

De acordo com Snell e Bohlander (2009), no processo de levantamento de necessidades de treinamento, é preciso analisar a empresa, a tarefa e a pessoa. No caso da UCX, observa-se que, ao identificar as necessidades de treinamento no âmbito da Empresa X, consideram-se, inicialmente, novos elementos do ambiente de negócios - tais como mercado consumidor, concorrência e tecnologia - os quais tenham relação com as estratégias adotadas pela organização.

Salienta-se que as ações de capacitação da UCX voltam-se, em especial, para o desenvolvimento das competências individuais consideradas necessárias ao adequado aproveitamento das soluções de ERP oferecidas pela Empresa X. Sob essa perspectiva, as características do cargo e as funções a ele atreladas são, então, identificadas e analisadas, com o propósito de estabelecer os conhecimentos e habilidades requeridos na atuação das pessoas. Nessa etapa, são levados em conta, também, os conhecimentos considerados essenciais ao alcance das metas da empresa.
Por fim, verifica-se a UCX procura identificar necessidades individuais de capacitação, relacionadas a conhecimentos individuais e específicos, as quais poderão ser atendidas por meio de treinamentos.

\subsubsection{Elaboração do projeto de treinamento}

Snell e Bohlander (2009) enfatizam que um aspecto importante para promover a ligação entre o colaborador e a organização é considerar, plenamente, os princípios psicológicos da aprendizagem, ou seja, levar em conta programas de treinamento cujas características ajudem os colaboradores a captar novos materiais, a entender como podem usá-los em suas vidas e a transferi-los à execução de suas atividades no trabalho. No caso da UCX, existem alguns princípios de aprendizagem como, por exemplo, estabelecimento de metas, modelagem para demonstrar comportamento, adaptação do treinamento ao estilo de cada pessoa, prática ativa, aprendizado do todo ou em partes, feedback e reforço.

$\mathrm{Na}$ construção do projeto de treinamento, os recursos didáticos compreendem videoaulas, áudios, textos explicativos, estudos de caso e exercícios práticos que simulam situações reais da rotina de uma empresa. Além do tutor que atua à distância, o qual é responsável, principalmente, por orientar os participantes no desenvolvimento das atividades do curso, têm-se os instrutores que conduzem as sessões presenciais de treinamento.

Nesse sentido, o design instrucional dos treinamentos é feito com foco no desenvolvimento das competências, necessárias ao pleno aproveitamento da solução de ERP. Para tal, ainda, o conteúdo instrucional busca propiciar aos participantes a aquisição dos principais conceitos de gestão empresarial, mediante sua aplicação feita no ERP. Salienta-se, contudo, que, além das competências técnico-funcionais, os treinamentos buscam, também, estimular o desenvolvimento de competências comportamentais, em consonância com os valores da Empresa X.

\subsubsection{Definição dos métodos de treinamento}

A Empresa $\mathrm{X}$ tem instituída uma prática de desenvolvimento funcional de seus colaboradores, cujos princípios - descritos a seguir - são adotados pela UCX:

a) plano de formação por função, o que significa que cada cargo existente na organização possui um plano específico para treinamento, o qual contempla um conjunto de cursos cujo conteúdo abrange o conhecimento 
necessário para a execução das atribuições previstas para o cargo;

b) grau de conhecimento escalonável em níveis que vão de 1 a 6, de forma que o plano de formação é distribuído em vários níveis com o objetivo de permitir a aquisição do conhecimento de forma gradativa;

c) treinamento em duas fases, sendo a primeira a $\mathrm{EaD}$ e a segunda presencial;

d) acompanhamento da efetividade do treinamento, por meio do acompanhamento de indicadores da qualidade dos serviços prestados pelos colaboradores.

Nesse sentido, em relação à metodologia adotada para a realização dos treinamentos na UCX, os treinamentos são realizados em três etapas. Na primeira, realizada via $\mathrm{EaD}$, por meio de videoaulas, manuais didáticos, atividades diversas no ambiente virtual e exercícios dirigidos, o participante direciona o estudo de acordo com o plano de formação de seu cargo, com prazos sugeridos pela UCX e acordados com seu superior imediato. Ao final, é feita a avaliação dos resultados de aprendizagem alcançados.

A segunda etapa envolve a simulação da implantação do ERP, em que o participante recebe um estudo de caso de uma empresa fictícia e simula a implantação do ERP em um sistema de treinamento. Como atividade complementar, o participante acompanha a implantação real do ERP em uma empresa cliente, pois se considera que a vivência em campo, de situações reais, leva ao aprofundamento do aprendizado. Também, julga-se que, ao participar como observador, o participante tem a possibilidade de desenvolver atitudes e comportamentos importantes para a ação do profissional em campo.

$\mathrm{Na}$ terceira etapa, em que o treinamento é presencial, faz-se uma nova simulação de implantação do ERP, em duas fases. Na primeira, com o acompanhamento do instrutor, por meio de dinâmicas que simulam as funções do sistema e informam sobre os processos e rotinas de trabalho. Na segunda fase, o participante simula outra implantação do ERP, contudo, sem o suporte do instrutor e sem consulta aos materiais de apoio. Após a aprovação nessa etapa, o participante é então, certificado na implantação do ERP da Empresa X.

Chiavenato (2009) faz classificações dos métodos de treinamento quanto ao uso, tempo e local. Quanto ao uso, observa-se que a UCX prioriza técnicas orientadas ao conteúdo, pois o treinamento tem como objetivo a transmissão de conhecimento. No que diz respeito ao tempo, a metodologia de treinamento pode ser aplicada tanto antes do ingresso no trabalho quanto após o ingresso. No caso da UCX, verifica-se o uso das duas formas, uma vez o treinamento no ERP é, também, aberto a profissionais que têm interesse em atuar na Empresa $X$, contudo ainda não estão participando de processo seletivo. Por fim, sobre o local, nota-se que os treinamentos da UCX possuem duas dimensões, uma interna à organização e outra externa, como ocorre na atividade de campo em que o participante acompanha a implantação do ERP em uma empresa cliente.

Snell e Bohlander (2009) também classificam os métodos de treinamento, pelo que chamam a atenção para a utilidade da $\mathrm{EaD}$, a qual é aplicada na primeira etapa dos treinamentos realizados pela UCX. Acredita-se que essa modalidade contribui para o envolvimento do participante, uma vez que ele terá autonomia para organizar-se em relação ao processo de ensino e aprendizagem, o que favorece o estabelecimento de um ambiente propício ao aprendizado. Ademais, julga-se que a modalidade exige o desenvolvimento de capacidades comportamentais pelo participante, como definição de metas, planejamento do tempo, disciplina e autoconfiança.

Ainda em relação aos métodos adotados, destaca-se o estudo de caso (SNELL; BOHLANDER, 2009), o qual, na UCX, é configurado na forma de uma simulação de implantação do ERP. Sua importância decorre do potencial do método em propiciar condições para a solução de problemas e tomada de decisão, o que leva ao aperfeiçoamento das capacidades de raciocínio crítico, flexibilidade e interação pessoal dos participantes.

\subsubsection{Implementação e avaliação do programa de treinamento}

Conforme indica Castro (2002), as atividades de implementação do programa de treinamento envolvem o gerenciamento de espaço físico e de materiais, a coordenação das pessoas envolvidas e procedimentos de comunicação e divulgação do programa. No caso da UCX, observa-se que, além dessas atividades, a implementação dos programas de treinamento abrange um processo contínuo de monitoramento dos resultados parciais, alcançados pelos participantes, no treinamento.

Em relação à avaliação de suas ações de treinamento, a UCX leva em conta, além dos resultados parciais e finais obtidos pelos participantes, a evolução dos indicadores de desempenhos destes, após a conclusão dos 
treinamentos. Os principais indicadores considerados são aqueles que evidenciam o quanto o colaborador aciona o suporte técnico da área de Help-Desk do ERP, o seu nível de produtividade, o nível de qualidade das tarefas executadas e o índice de satisfação dos clientes atendidos pelo colaborador.

Ainda que, como apontam Overcast et al. (2009), a mensuração dos resultados de um treinamento mostra-se um procedimento complexo, verifica-se que a UCX analisa os aspectos essenciais relativos aos resultados da ação, em consonância com Snell e Bohlander (2009).

Com base no exposto, considera-se que o processo de treinamento desenvolvido pela UCX possui as características essenciais da abordagem sistêmica de treinamento, em que se observam as etapas de levantamento de necessidades de treinamento, elaboração do projeto de treinamento, definição dos métodos de treinamento e de implementação e avaliação do projeto.

Em consonância com a visão expressada pelos gestores da UCX, julga-se que tal metodologia contribui para a capacidade apresentada por essa UC de desenvolver ações de treinamento que proporcionam aprendizados efetivos aos seus participantes e alinhados aos objetivos estratégicos da Empresa X.

Não obstante, nota-se que as ações de treinamento desenvolvidas pela UCX estão fortemente baseadas na $\mathrm{EaD}$, o que, apesar de dentre os métodos de treinamento encontrarem-se atividades de campo e capacitações presenciais, pode orientar o foco dos treinamentos para a aquisição de informações e competências técnico-funcionais. Isto porque as ações de treinamento baseadas em $\mathrm{EaD}$ mostram-se, muitas vezes, limitadoras da interação e cooperação entre os participantes, as quais favorecem a aprendizagem comportamental baseada na comunicação e compartilhamento de experiências entre os participantes.

\section{Considerações finais}

Nesta pesquisa objetivou-se analisar o processo de treinamento desenvolvido pela UCX, a unidade de educação corporativa de uma grande indústria desenvolvedora de softwares de gestão empresarial, a Empresa X, com o propósito de discutir de que forma esse processo contém elementos e características da abordagem sistêmica de treinamento.

Os resultados mostram que os programas de trei- namento da UCX são desenvolvidos em consonância com a abordagem sistêmica de treinamento, o que, se julga, contribui para elevar o nível de efetividade da aprendizagem dos participantes. Avalia-se, também, que, ao levar em conta as etapas de desenvolvimento de um projeto de treinamento propostas pela abordagem sistêmica, as ações de treinamento da UCX mostram-se alinhadas aos objetivos estratégicos da Empresa X.

Contudo, ainda que os benefícios do uso de uma abordagem sistêmica de treinamento se mostrem presentes, considera-se que, pelo fato de seus programas serem significativamente baseados na EaD, cuja dinâmica oferece poucas oportunidades para a interação entre os participantes, é preciso que a UCX leve em conta novas estratégias de aprendizagem, voltadas ao desenvolvimento de competências comportamentais pelos participantes. Tais estratégias podem basear-se na modalidade $\mathrm{EaD}$, por meio da utilização de recursos como videoconferências e resolução de problemas em tempo real.

Salienta-se que esta pesquisa possui, como principal limitação, a impossibilidade de generalização de seus resultados, uma vez que a análise e discussão do objeto de pesquisa basearam-se, sobremaneira, na visão dos gestores entrevistados, ou seja, apenas uma fonte. Ademais, a organização pesquisada foi selecionada por conveniência, não representando, assim, a diversidade do universo de organizações brasileiras.

Partindo-se dos resultados encontrados, sugere-se a realização de novos estudos, focados na compreensão da inter-relação entre as abordagens, modalidades e métodos de treinamento adotados pela UC e os resultados em termos de aprendizagem dos participantes e/ou do desempenho corporativo. Nesse caso, seria adequado que os dados fossem coletados em diversas UCs, para possibilitar a comparação dos resultados, especialmente por setor de atividade.

\section{Referências}

ALPERSTEDT, C. Universidades corporativas: discussão e proposta de uma definição. Revista de Administração Contemporânea, Curitiba, v. 5, n. 3, 2001.

BOOG, G. Os novos paradigmas do mundo dos negócios. In: BOOG, G.; BOOG, M. (Coord.). Manual de treinamento e desenvolvimento. São Paulo: Pearson Education, 2007. 
BRANDÃO, G. R. Gestão de pessoas e as universidades corporativas: dois lados da mesma moeda? Revista de Administração de Empresas, v. 46, n. 2, p. 22-33, 2006.

BRYMAN, A. Social research methods. 3. ed. Oxford: Oxford University Press, 2004.

CARVAlHO, A. V. Funções básicas do sistema de RH: atrair, escolher e preparar. Rio de Janeiro: Qualitymark, 2007.

CARVAlHO, A. V.; NASCIMENTO, L. P.; SERAFIM, O. C. G. Administração de recursos humanos. São Paulo: Cengage Learning, 2012.

CASTRO, A. P. Avaliação e validação do treinamento. In: BOOG, G.; BOOG, M. (Coord.). Manual de gestão de pessoas e equipes. São Paulo: Gente, 2002.

CHIAVENATO, I. Gestão de pessoas: o novo papel dos recursos humanos nas organizações. Rio de Janeiro: Campus, 2009.

CRESWELL, J. W. Projeto de pesquisa: métodos qualitativo, quantitativo e misto. 2. ed. Porto Alegre: Artmed, 2010.

EBOLI, M. O desenvolvimento das pessoas e a educação corporativa. In: FLEURY, M. T. L. As pessoas na organização. São Paulo: Gente, 2002.

FLICK, U. Introdução à pesquisa qualitativa. 3. ed. Porto Alegre: Artmed, 2009.

HOLLAND, P.; PYMAN, A. Corporate universities: a catalyst for strategic human resource development? Journal of European Industrial Training, v. 30, n. 1, p. 19-31, 2006.

KRUMM, D. Psicologia do trabalho. São Paulo: LTC, 2005.

MEISTER, J. Educação corporativa: a gestão do capital intelectual através das universidades corporativas. São Paulo: Makron Books, 1999.
MERRIAM, S. B. Qualitative research in practice: examples for discussion and analysis. San Francisco: Jossey-Bass, 2002.

MILKOVICH, G. T.; BOUDREAU, J. W. Administração de recursos humanos. São Paulo: Atlas, 2000.

MORIN, L.; RENAUD, S. Participation in corporate university training: its effect on individual job performance. Canadian Journal of Administrative Sciences, v. 21, n. 4, p. 295-306, 2004.

MUNDIM, A. P. F. Desenvolvimento de produtos e educação corporativa. São Paulo: Atlas, 2002.

OVERCAST, S. et al. A case example of assessment and evaluations: building capability in a corporate university. Performance Improvement, v. 48, n. 6, p. 5-15, 2009.

SANTOS, Antônio Raimundo dos. Metodologia científica: a construção do conhecimento. Rio de Janeiro: DP\&A, 1999.

SHAM, C. An exploratory study of corporate universities in China. Journal of Workplace Learning, v. 19, n. 4, p. 257-264, 2007.

SILVA, M. E. Relações entre impacto do treinamento no trabalho e estratégia empresarial: o caso da Eletronorte. Revista de Administração Contemporânea, v. 10, n. 3, 2006.

SNELL, S.; BOHLANDER, G. Administração de recursos humanos. São Paulo: Cengage Learning, 2009.

TOLEDO, F.; MILIONI, B. Dicionário de recursos humanos. 3. ed. São Paulo: Atlas, 1986. 